This is a preprint version of the following article:

Brey, P. (2008). 'Virtual Reality and Computer Simulation,' Ed. Himma, K. and Tavani, H., Handbook of Information and Computer Ethics, John Wiley \& Sons.

\title{
Virtual Reality and Computer Simulation
}

\section{Introduction}

Virtual reality and computer simulation have not received much attention from ethicists. It is argued in this essay that this relative neglect is unjustified, and that there are important ethical questions that can be raised in relation to these technologies. First of all, these technologies raise important ethical questions about the way in which they represent reality and the misrepresentations, biased representations and offensive representations that they may contain. In addition, actions in virtual environments can be harmful to others, and can also be morally problematic from the point of view of deontological and virtue ethics. Although immersive virtual reality systems are not yet used on a large scale, nonimmersive virtual reality is regularly experienced by hundreds of millions of users, in the form of computer games and virtual environments for exploration and social networking. These forms of virtual reality also raise ethical questions regarding their benefits and harms to users and society, and the values and biases contained in them.

This paper has the following structure. The next section will describe what virtual reality and computer simulations are and what the current applications of these technologies are. This is followed by a section that analyzes the relation between virtuality and reality, and asks whether virtuality can and should function as a substitute for reality. Three subsequent sections discuss ethical aspects of representation in virtual reality and computer simulations, the ethics of behavior in virtual reality and the ethics of computer games. A concluding section discusses issues of professional ethics in the development and professional use of virtual reality systems and computer simulations.

\section{Background: The Technology and Its Applications}

\section{Virtual reality}

Virtual reality (VR) technology emerged in the 1980s, with the development and marketing of systems consisting of a head mounted display (HMD) and datasuit or dataglove attached to a computer. These technologies simulated three-dimensional (3-D) environments displayed in surround stereoscopic vision on the head mounted display. The user could navigate and interact with simulated environments through the datasuit and dataglove, items that tracked the positions and motions of body parts and allowed the computer to modify its output depending on the recorded positions. This original technology has helped define what is often meant by "virtual reality": an immersive, 
interactive three-dimensional computer-generated environment in which interaction takes place over multiple sensory channels and includes tactile and positioning feedback.

According to Sherman and Craig (2003), there are four essential elements in virtual reality: a virtual world, immersion, sensory feedback, and interactivity. A virtual world is a description of a collection of objects in a space and rules and relationships governing these objects. In virtual reality systems, such virtual worlds are generated by a computer. Immersion is the sensation of being present in an environment, rather than just observing an environment from the outside. Sensory feedback is the selective provision of sensory data about the environment based on user input. The actions and position of the user provide a perspective on reality and determines what sensory feedback is given. Interactivity, finally, is the responsiveness of the virtual world to user actions.

Interactivity includes the ability to navigate virtual worlds and to interact with objects, characters and places.

These four elements can be realized to a greater and lesser degree with a computer, and that is why there are both broad and narrow definitions of virtual reality. A narrow definition would only define fully immersive and fully interactive virtual environments as VR. However, there are many virtual environments that do not meet all these criteria to the fullest extent possible, but that could still be categorized as VR. Computer games played on a desktop with a keyboard and mouse, like Doom and HalfLife, are not fully immersive, and sensory feedback and interactivity in them is more limited than in immersive VR systems that include a head mounted display and datasuit. Yet, they do present virtual worlds that are immersive to an extent, and that are interactive and involve visual and auditory feedback. In Brey (1999) I therefore proposed a broader definition of virtual reality, as a three-dimensional interactive computergenerated environment that incorporates a first-person perspective. This definition includes both immersive and nonimmersive (screen-based) forms of VR.

The notion of a virtual world, or virtual environment, as defined by Sherman and Craig, is broader than that of virtual reality. A virtual world can be realized by means of sensory feedback, in which case it yields virtual reality, but it can also be realized without it. Classical text-based adventure games like Zork, for example, play in interactive virtual worlds, but users are informed about the state of this world through text. They provide textual inputs, and the game responds with textual information rather than sensory feedback about changes in the world. A virtual world is hence an interactive computer-generated environment, and virtual reality is a special type of virtual world that involves location- and movement-relative sensory feedback.

Next to the term "virtual reality," there is the term "virtuality" and its derivative adjective "virtual". This term has a much broader meaning than the term "virtual reality" or even "virtual environment". As explained more extensively in the next section, the term "virtual" refers to anything that is created or carried by a computer and that mimics a "real", physically localized entity, as in "virtual memory" and "virtual organization". In this essay, the focus will be on virtual reality and virtual environments, but occasionally, especially in the next section, the broader phenomenon of virtuality will be discussed as well.

Returning to the topic of virtual reality, a distinction can be made between singleuser and multi-user or networked VR. In single-user VR, there is only one user, whereas in networked VR, there are multiple users who share a virtual environment and appear to 
each others as avatars, which are graphical representations of the characters played by users in VR. A special type of VR is augmented reality, in which aspects of simulated virtual worlds are blended with the real world that is experienced through normal vision or a video link, usually through transparent glasses on which computer graphics or data are overlaid. Related to VR, furthermore, are telepresence and teleoperator systems, systems that extend a person's sensing and manipulation capability to a remote location by displaying images and transmitting sounds from a real environment that can (optionally) be acted on from a distance through remote handling systems such as robotic arms.

\section{Computer simulation}

A computer simulation is a computer program that contains a model of a particular system (either actual or theoretical) and that can be executed, after which the execution output can be analyzed. Computer simulation is also the name of the discipline in which such models are designed, executed and analyzed. The models in computer simulations are usually abstract and either are or involve mathematical models. Computer simulation has become a useful part of the mathematical modeling of many natural systems in the natural sciences, human systems in the social sciences, and technological systems in the engineering sciences, in order to gain insight into the operations of these systems and to study the effects of alternative conditions and courses of action.

It is not usually an aim in computer simulations, as it is in virtual reality, to do realistic visual modeling of the systems that they simulate. Some of these systems are abstract, and even for those systems that are concrete, the choice is often made not to design graphic representations of the system, but to rely solely on abstract models of it. When graphical representations of concrete systems are used, they usually only represent features that are relevant to the aims of the simulation, and do not aspire to the realism and detail aspired to in virtual reality.

Another difference with virtual reality is that computer simulations need not be interactive. Usually, simulators will determine a number of parameters at the beginning of a simulation and then "run" the simulation without any interventions. In this standard case, the simulator is not himself defined as part of the simulation, as would happen in virtual reality. An exception is an interactive simulation, which is a special kind of simulation, also referred to as a human-in-the-loop simulation, in which the simulation includes a human operator. An example of such a simulation would be a flight simulator. If a computer simulation is interactive and makes use of three-dimensional graphics and sensory feedback, it also qualifies as a form of virtual reality. Sometimes, also, the term "computer simulation" is used to include any computer program that models a system or environment, even if it is not used to gain insight into the operation of a system. In that broad sense, virtual environments, at least those that aim to do realistic modeling, would also qualify as computer simulations.

\section{Applications}

VR is used to simulate both real and imaginary environments. Traditional VR applications are found in medicine, education, arts and entertainment, and the military 
(Burdea and Coiffet, 2003). In medicine, VR is used for the simulation of anatomical structures and medical procedures in education and training, for example for performing virtual surgery. Increasingly, VR is also being used for (psycho)therapy, for instance for overcoming anxiety disorders by confronting patients with virtual anxiety-provoking situations (Wiederhold and Wiederhold, 2004). In education, VR is used in explorationbased learning and learning by building virtual worlds. In the arts, VR is used to create new art forms and to make the experience of existing art more dynamic and immersive. In entertainment, mostly nonimmersive, screen-based forms of VR are used in computer and video games and arcades. This is a form of VR that many people experience on a regular basis. In the military, finally, VR is used in a variety of training contexts for army, navy and air force. Emerging applications of VR are found in manufacturing, architecture, and training in a variety of (dangerous) civilian professions.

Computer simulations are used in the natural and social sciences to gain insight into the functioning of natural and social systems, and in the engineering sciences for performance optimization, safety engineering, training and education. They are used on a large scale in the natural and engineering sciences, where such fields have sprung up as computational physics, computational neuroscience, computational fluid mechanics, computational meteorology and artificial life. They are also used on a somewhat more modest scale in the social sciences, for example in the computational modeling of cognitive processes in psychology, in the computational modeling of artificial societies and social processes, in computational economic modeling, and in strategic management and organizational studies. Computer simulations are increasingly used in education and training, to familiarize students with the workings of systems and to teach them to interact successfully with such systems.

\section{Virtuality and Reality}

\section{The Distinction between the Virtual and the Real}

In the computer era, the term "virtual" is often contrasted with "real". Virtual things, it is often believed, are things that only have a simulated existence on a computer, and are therefore not real, like physical things. Take, for example, rocks and trees in a virtual reality environment. They may look like real rocks and trees, but we know that they have no mass, no weight, and no identifiable location in the physical world, and are just illusions generated through electrical processes in microprocessors and the resulting projection of images on a computer screen. "Virtual" hence means "imaginary", "makebelieve", "fake", and contrasts with "real", "actual" and "physical". A virtual reality is therefore always only a make-believe reality, and can as such be used for entertainment or training, but it would be a big mistake, in this view, to call anything in virtual reality real, and to start treating it as such.

This popular conception of the contrast between virtuality and reality can, however, be demonstrated to be incorrect. "Virtual" is not the perfect opposite of "real", and some things can be virtual and real at the same time. To see how this is so, let us start by considering the semantics of "virtual". The word "virtual" has two traditional, 
pre-computer meanings. On the first, most salient meaning, it refers to things that have certain qualities in essence or in effect, but not in name. For instance, if only a few buffalo are left, one can say that buffalo are virtually extinguished, extinguished for all practical purposes, even though they are not formally or actually extinguished. Virtual can also mean imaginary, and therefore not real, as in optics, where reference is made to virtual foci and images. Notice that only on the second, least salient meaning, "virtual" contrasts with "real". On the more salient meaning, it does not mean "unreal" but rather "practically but not formally real".

In the computer era, the word "virtual" came to refer to things simulated by a computer, like virtual memory, which is memory that is not actually built into a processor, but nevertheless functioning as such. Later, the scope of the term "virtual" has expanded to include anything that is created or carried by a computer and that mimics a "real" equivalent, like a virtual library and a virtual group meeting. The computer-based meaning of "virtual" conforms more with the traditional meaning of "virtual" as "practically but not formally real" than with "unreal". Virtual memory, for example, is not unreal memory, but rather a simulation of physical memory that can effectively function as real memory.

Under the above definition of "virtual" as "created or carried by a computer and mimicking a "real" equivalent," virtual things and processes are imitations of real things, but this need not also preclude them from being real themselves. A virtual game of chess, for example, is also a real game of chess. It is just not played with a physically realized board and pieces. I have argued (Brey, 2003) that a distinction can be made between two types of virtual entities: simulations and ontological reproductions.

Simulations are virtual versions of real-world entities that have a perceptual or functional similarity to them, but that do not have the pragmatic worth or effects of the corresponding real-world equivalent. Ontological reproductions are computer imitations of real-world entities that have (nearly) the same value or pragmatic effects as their real world counterparts. They hence have a real-world significance that extends beyond the domain of the virtual environment and that is roughly equal to that of their physical counterpart.

To appreciate this contrast, consider the difference between a virtual chess game and a virtual beer. A virtual beer is necessarily a mere simulation of a real beer: it may look much like a real one, and may be lifted and consumed in a virtual sense, but it does not provide the taste and nourishment of a real beer and will never get one drunk. A virtual chess game, in contrast, may lack the physical sensation of moving real chess pieces on a board, but this sensation is considered peripheral to the game, and in relevant other respects, playing virtual chess is equivalent to playing chess with physical pieces. This is not to say that the distinction between simulations and ontological reproductions is unproblematic; it is ultimately a pragmatic distinction, and a virtual entity will be classified as one or the other depending on whether it is judged to share enough of the essential features of its physical counterpart.

In Brey (2003), I argued that two classes of physical objects and processes can be ontologically reproduced on computers. A first class consists of physical entities that are defined in terms of visual, auditory or computational properties that can be fully realized on multimedia computers. Such entities include images, movies, musical pieces, stereo 
systems and calculators, which are all such that a powerful computer can successfully reproduce their essential physical or formal properties.

A second class consists of what John Searle (1995) has called institutional entities, which are entities that are defined by a status or function that has been assigned to them within a social institution or practice. Examples of institutional entities are activities like buying, selling, voting, owning, chatting, playing chess, trespassing and joining a club, and requisite objects like contracts, money, letters and chess pieces. Most institutional entities are not dependent on a physical medium, because they are only dependent on the collective assignment of a status or function. For instance, we call certain pieces of paper money not because of their inherent physical nature but because we collectively assign monetary value to them. But we could also decide, and have decided, to assign the same status to certain sequences of bits that float around on the Internet. In general, if an institutional entity exists physically, it can also exist virtually. Therefore, many of our institutions and institutional practices, whether social, cultural, religious or economic, can exist in virtual or electronic form.

It can be concluded that many virtual entities can be just as real as their physical counterparts. Virtuality and reality are therefore not each others opposites. Nevertheless, a large part of ordinary reality, that includes most physical objects and processes, cannot be ontologically reproduced in virtual form. In addition, institutional virtual entities can both possess and lack real-world implications. Sometimes virtual money can also be used as real money, whereas at other times, it is only a simulation of real money. People can also disagree on the status of virtual money, with some accepting it as legal tender, and others distrusting it. The ontological distinction between reality and virtuality is for these reasons confusing, and the ontological status of encountered virtual objects will often be not immediately clear.

\section{Is the Distinction Disappearing?}

Some authors have argued that the emergence of computer-generated realities is working to erase the distinction between simulation and reality, and therefore between truth and fiction. Jean Baudrillard (1995), for example, has claimed that information technology, media, and cybernetics have yielded a transition from an era of industrial production to an era of simulation, in which models, signs and codes mediate access to reality and define reality to the extent that it is no longer possible to make any sensible distinction between simulations and reality, so that the distinction between reality and simulation has effectively collapsed. Similarly, Albert Borgmann (1999) has argued that virtual reality and cyberspace have lead many people to confuse them for alternative realities that have the same actuality of the real world, thus leading to a collapse of the distinction between representation and reality, whereas according to him VR and cyberspace are merely forms of information and should be treated as such.

Philip Zhai (1998), finally, has argued that there is no principled distinction between actual reality and virtual reality, and that with further technological improvements in VR, including the addition of functional teleoperation, virtual reality could be made totally equivalent to actual reality in its functionality for human life. Effectively, Zhai is arguing that any real-world entity can be ontologically reproduced in 
VR given the right technology, and that virtual environments are becoming ontologically more like real environments as technology progresses.

Are these authors right that the distinction between virtuality and reality, and between simulation and reality, is disappearing? First, it is probably true that there is increasingly less difference between the virtual and the real. This is because, as has already been argued, many things are virtual and real at the same time. Moreover, the number of things that are both virtual and real seem to be increasing. This is because as the possibilities of computers and computer networks increase, more and more physical and institutional entities are reproduced in virtual form. There is a flight to the digital realm, in which many believe it is easier and more fun to buy and sell, listen to music or look at art, or do your banking. For many people, therefore, an increasingly large part of their real lives is also virtual, and an increasingly large part of the virtual is also real.

Even if virtuality and reality are not opposite concepts, simulation and reality, and representation and reality, certainly are. Are these two distinctions disappearing as well? Suggesting that they at least become more problematic is the fact that more and more of our knowledge of the real world is mediated by representations and simulations, whether they are models in science, raw footage and enactments in broadcast news, and stories and figures in newspapers or on the Internet. Often, it is not possible, in practice or in principle, to verify the truth or accuracy of these representations through direct inspection of the corresponding state-of-affairs. Therefore, one might argue that these representations become reality for us, for they are all the reality we know.

In addition, the distinction between recordings and simulations is becoming more difficult to make. Computer technology has made it easy to manipulate photos, video footage and sound recordings, and to generate realistic imagery, so that it is nowadays often unclear of photographic images or video footage on the Internet or in the mass media whether they are authentic or fabricated or enacted. The trend in mass media towards "edutainment" and the enactment and staging of news events has further problematized the distinction.

Yet, all this does not prove that the distinction between simulation/representation and reality has collapsed. People do not get all of their information from media representations. They also move around and observe the world for themselves. People still question and critically investigate whether representations are authentic or correspond to reality. People hence still maintain an ontological distinction, even though it has become more difficult epistemologically to discern whether things and events are real or simulated. Zhai's suggestion that the distinction could be completely erased through further perfection of virtual reality technology is unlikely to hold because it is unlikely that virtual reality could ever fully emulate actual reality in its functionality for human life. Virtual reality environments cannot, after all, sustain real biological processes, and therefore they can never substitute for the complete physical world.

\section{Evaluating the Virtual as a Substitute for the Real}

Next to the ontological and epistemological questions regarding distinction between the virtual and the real and how we can know this distinction, there is the normative question of how we should evaluate virtuality as a substitute for reality. First of all, are virtual things better or worse, more or less valuable, than their physical counterparts? Some 
authors have argued that they are in some ways better: they tend to be more beautiful, shiny and clean, and more controllable, predictable, and timeless. They attain, as Michael Heim (1983) has argued, a supervivid hyper-reality, like the ideal forms of platonism, more perfect and permanent than the everyday physical world, answering to our desire to transcend our mortal bodies and reach a state of permanence and perfection. Virtual reality, it may seem, can help us live lives that are more perfect, more stimulating and more in accordance with our fantasies and dreams.

Critics of virtuality have argued that the shiny, polished objects of VR are mere surrogates: simplified and inferior substitutes for reality that lack authenticity. Albert Borgmann (1999), for example, has argued that virtuality is an inadequate a substitute for reality, because of its fundamental ambiguity and fragility, and lacks the engagement and splendor of reality. He also argues that virtuality threatens to alter our perspective on reality, causing us to see it as yet another sign or simulation. Hubert Dreyfus (2001) has argued that presence in VR and cyberspace gives a disembodied and therefore false experience of reality and that even immersive VR and telepresence present one with impoverished experiences.

Another criticism of the virtual as a substitute for the real is that investments in virtual environments tend to correlate with disinvestments in people and activities in real life (Brey, 1998). Even if this were to be no loss to the person making the

disinvestments, it may well be a loss to others affected by it. If a person takes great effort in caring for virtual characters, he or she may have less time left to give similar care and emotional attention to actual persons and animals, or may be less interested in giving it. In this way, investments in VR could lead to a neglect of real life and therefore a more solitary society. On the other hand, virtual environments can also be used to vent aggression, harming only virtual characters and property and possibly preventing similar actions in real life.

\section{Representation and Simulation: Ethical Issues}

VR and computer simulations are representational media: they represent real or fictional objects and events. They do so by means of different types of representations: pictorial images, sounds, words and symbols. In this section, ethical aspects of such representations will be investigated. It will be investigated whether representations are morally neutral and whether their manufacture and use in VR and computer simulations involves ethical choices.

\section{Misrepresentations, Biased Representations and Indecent Representations}

I will argue that representations in VR or computer simulations can become morally problematic for any of three reasons. First, they may cause harm by failing to uphold standards of accuracy. That is, they may misrepresent reality. Such representations will be called misrepresentations. Second, they may fail to uphold standards of fairness, thereby unfairly disadvantaging certain individuals or groups. Such representations will be called biased representations. Third, they may violate standards of decency and public morality. I will call such representations indecent representations. 
Misrepresentation in VR and computer simulation occurs when it is part of the aim of a simulation to realistically depict aspects of the real world, yet the simulation fails to accurately depict these features (Brey, 1999). Many simulations aim to faithfully depict existing structures, persons, state-of-affairs, processes or events. For example, VR applications have been developed that simulate in great detail the visual features of existing buildings such as the Louvre or Taj Mahal or the behavior of existing automobiles or airplanes. Other simulations do not aim to represent particular existing structures, but nevertheless aim to be realistic in their portrayal of people, things and events. For example, a VR simulation of military combat will often be intended to contain realistic portrayals of people, weaponry and landscapes without intending to represent particular individuals or a particular landscape.

When simulations aim to be realistic, they are expected to live up to certain standards of accuracy. These are standards that define the degree of freedom that exist in the depiction of a phenomenon, and that specify what kinds of features must be included in a representation for it to be accurate, what level of detail is required, and what kinds of idealizations are permitted. Standards of accuracy are fixed in part by the aim of a simulation. For example, a simulation of surgery room procedures should be highly accurate if it is used for medical training, somewhat accurate when sold as edutainment, and need not be accurate at all when part of a casual game. Standard of accuracy can also be fixed by promises or claims made by manufacturers. For example, if a game promises that surgery room procedures in it are completely realistic, the standards of accuracy for the simulation of these procedures will be high. People may also disagree about the standards of accuracy that are appropriate for a particular simulation. For example, a VR simulation of military combat that does not represent killings in graphic detail may be discounted as inaccurate and misleading by anti-war activists, but may be judged to be sufficiently realistic for the military for training purposes.

Misrepresentations of reality in VR and computer simulations are morally problematic to the extent that they can result in harm. The greater these harms are, and the greater the chance that they occur, the greater the moral responsibility of designers and manufacturers to ensure accuracy of representations. Obviously, inaccuracies in VR simulations of surgical procedures for medical training or computer simulations to test the bearing power of bridges can lead to grave consequences. A misrepresentation of the workings of an engine in educational software causes a lesser or less straightforward harm: it causes students to have false beliefs, some of which could cause harms at a later point in time.

Biased representations constitute a second category of morally problematic representations in VR modeling and computer simulation (Brey, 1999). A biased representation is a representation that unfairly disadvantages certain individuals or groups or that unjustifiably promotes certain values or interests over others. A representation can be biased in the way it idealizes or selectively represents phenomena. For example, a simulation of global warming may be accurate overall but unjustifiably ignore the contribution to global warming made by certain types of industries or countries. Representations can also be biased by stereotyping people, things and events. For example, a computer game may contain racial or gender stereotypes in its depiction of people and their behaviors. Representations can moreover be biased by containing implicit assumptions about the user, as in a computer game that plays out male 
heterosexual fantasies, thereby assuming that players will generally be male and heterosexual. They can also be biased by representing affordances and interactive properties in objects that make them supportive of certain values and uses but not of others. For example, a gun in a game may be designed so that it can used to kill but not to knock someone unconscious.

Indecent representations constitute a third and final category of morally problematic representations. Indecent representations are representations that are considered shocking or offensive or that are held to break established rules of good behavior or morality and that are somehow shocking to the senses or moral sensibilities.

Decency standards vary widely across different individuals and cultures however, and what is shocking or immoral to some will not be so to others. Some will find any depiction of nudity, violence or physical deformities indecent, whereas others will find any such depiction acceptable. The depiction of particular acts, persons or objects may be considered blasphemous in certain religions but not outside these religions. For this reason, the notion of an indecent representation is a relative notion, and there will usually be disagreement about what representations count as indecent. In addition, the context in which representation take place may also influence whether it is considered decent. For example, the representation of open heart surgery, with some patients surviving the procedure but others dying on the operation table, may be inoffensive in the context of a medical simulator, but offensive in the context of a game that makes light of such a procedure.

\section{Virtual Child Pornography}

Pornographic images and movies are considered indecent by many, but there is a fairly large consensus that people have a right to produce pornography and use it in private. Such a consensus does not consist for certain extreme forms of pornography, including child pornography. Child pornography is considered wrong because it harms the children that are used to produce it. But what about virtual child pornography? Virtual child pornography is the digital creation of images or animated pictures that depict children engaging in sexual activities or that depict them in a sexual way. Nowadays, such images and movies can be made to be highly realistic. No real children are abused in this process, and therefore the major reason for outlawing child pornography does not apply for it. Does this mean that virtual child porn is morally permissible and that its production and consumption should be legal?

The permissibility of virtual child porn has been defended on the argument that no actual harm is done to children and that that people have a right to free speech by which they should be permitted to produce and own virtual child pornography, even if others find such images offensive. Indeed, the U.S. Supreme Court struck down a congressional ban on virtual child porn in 2002 with the argument that this ban constituted too great a restriction on free speech. The court also claimed that no proof had been given of a connection between computer-generated child pornography and the exploitation of actual children. An additional argument that is sometimes used in favor of virtual child porn is that its availability to pedophiles may actually decrease the chances that they will harm children. 
Opponents of virtual child porn have sometimes responded with deontological arguments, claiming that it is degrading to children and undermines human dignity. Such arguments cut little ice, however, in a legal arena that is focused on individual rights and harms. Since virtual child porn does not seem to violate the individual rights, opponents have tried out various arguments to the effect that it does cause harm. One existing argument is that virtual child porn causes indirect harm to children because it encourages child abuse. This argument goes opposite the previously stated argument that virtual child porn should be condoned because it makes child abuse less likely. The problem is that it is very difficult to conduct studies that provide solid empirical evidence for either position. Another argument is that failing to criminalize virtual child porn will harm children because it makes it difficult to enforce laws that prohibit actual child pornography. This argument has been used often by law enforcers to criminalize virtual child porn. As Neil Levy (2002) has argued, this argument is however not plausible, amongst other reasons because experts are usually able to make the distinction between virtual and actual pictures.

Levy's own argument against virtual child porn is not that it will indirectly harm children, but that it may ultimately harm women by eroticizing inequality in sexual relationships. He admits, however, that he lacks the empirical evidence to back up this claim. Per Sandin (2004) has presented an argument with better empirical support, which is that virtual child porn should be outlawed because it causes significant harm to a great many people who are revulsed by it. The problem with this argument, however, is that it gives too much weight to harm caused by offense. If actions should be outlawed whenever they offend a large group of people, then individual rights would be drastically curtailed, and many things, ranging from homosexual behavior to interracial marriage, would still be illegal. It can be concluded that virtual child pornography will remain a morally controversial issue for some time to come, as no decisive arguments for or against it have been provided so far.

\section{Depiction of Real Persons}

Virtual environments and computer simulations increasingly include characters that are modeled after the likeness of real persons, whether living or deceased. Also, films and photographs increasingly include manipulated or computer-generated images of real persons who are placed in fictional scenes or are made to perform behaviors that they have not performed in real life. Such appropriations of likenesses are often made without the person's consent. Is such consent morally required, or should the depictions of real persons be seen as an expression of artistic freedom or free speech?

Against arguments for free speech, three legal and moral arguments have traditionally been given for restrictions on the use of someone's likeness (Tabach-Bank, 2004). First, the right to privacy has been appealed to. It has been argued that the right to privacy includes a right to live a life free from unwarranted publicity (Prosser, 1960). The public use of someone's likeness can violate someone's privacy by intruding upon his seclusion or solitude or into his private affairs, by working to publicly disclose embarrassing private facts about him, or to place him in a false light in the public eye. A second argument for restricting the use of someone's likeness is that it can be used for defamation. Depicting someone in a certain way, for example as being involved in 
immoral behavior or in a ridiculous situation, can defame him by harming his public reputation.

In some countries, like the U.S., there is also a separate recognized right of publicity. The right to publicity is an individual's right to control and profit from the commercial use of his name, likeness and persona. The right to publicity has emerged as a protection of the commercial value of the identity of public personalities, or celebrities, who frequently use their identity to sell or endorse products or services. It is often agreed that celebrities have less of an expectation of privacy, because they are public personalities, but have a greater expectation of a right to publicity. In the use of the likenesses of real persons in virtual environments or doctored digital images, rights to free speech, freedom of the press and freedom of artistic expression will therefore have to be balanced against the right to privacy, the right of publicity and the right to protection from defamation.

\section{Behavior in Virtual Environments: Ethical Issues}

The preceding section focused on ethical issues in design and embedded values in VR and computer simulations. This section focuses on ethical issues in the use of VR and interactive computer simulations. Specifically, the focus will be on the question whether actions within the worlds generated by these technologies can be unethical. This issue will be analyzed for both single-user and multi-user systems. Before it will be taken up, it will first be considered how actions in virtual environments take place, and what the relation is between users and the characters as which they appear in virtual environments.

\section{Avatars, Agency and Identity}

In virtual environments, users assume control over a graphically realized character called an avatar. Avatars can be built after the likeness of the user, but more often, they are generic persons or fantasy characters. Avatars can be controlled from a first-person perspective, in which the user sees the world through the avatar's eyes, or from a thirdperson perspective. In multi-user virtual environments, there will be multiple avatars corresponding to different users. Virtual environments also frequently contain bots, which are programmed or scripted characters that behave autonomously and are controlled by no one.

The identity that users assume in a virtual environment is a combination of the features of the avatar they choose, the behaviors that they choose to display with it, and the way others respond to the avatar and its behaviors. Avatars can function as a manifestation of the user, who behaves and acts like himself, and to whom others respond as if it is the user himself, or as a character that has no direct relation to the user and that merely plays out a role. The actions performed by avatars can therefore range from authentic expressions of the personality and identity of the user to experimentation with identities that are the opposite of who the user normally is.

Whether or not the actions of an avatar correspond with how a user would respond in real life, there is no question that the user is causally and morally responsible for actions performed by his or her avatar. This is because users normally have full 
control over the behavior of their avatars through one or more input devices. There are occasional exceptions to this rule, because avatars are sometimes taken over by the computer and then behave as bots. The responsibility for the behavior of bots could be assigned to either their programmer or to whomever introduced them into a particular environment, or even to the programmer of the environment for not disallowing harmful actions by bots (Ford, 2001).

\section{Behavior in Single-User VR}

Single-user VR offers much less possibilities for unethical behavior than multi-user VR, because there are no other human beings that could be directly affected by the behavior of a user. The question is if there are any behaviors in single-user VR that could qualify as unethical. In Brey (1999), I considered the possibility that certain actions that are unethical when performed in real life could also be unethical when performed in singleuser VR. My focus was particularly on violent and degrading behavior towards virtual human characters, such as murder, torture and rape. I considered two arguments for this position, the argument from moral development and the argument from psychological harm.

According to the argument from moral development, it is wrong to treat virtual humans cruelly because doing so will make it more likely that we will treat real humans cruelly. The reason for this is that the emotions appealed to in the treatment of virtual humans are the same emotions that are appealed to in the treatment of real humans, because these actions resemble each other so closely. This argument has recently gained empirical support (Slater et al., 2006). The argument from psychological harm is that third parties may be harmed by the knowledge or observation that people engage in violent, degrading or offensive behavior in single-user VR and that therefore this behavior is immoral. This argument is similar to the argument attributed to Sandin in my earlier discussion of indecent representations. I claimed in Brey (1999) that although harm may be caused by particular actions in single-user VR because people may be offended by them, it does not necessarily follow that the actions are immoral, but only that they cause indirect harm to some people. One would have to balance such harms against any benefits, such as pleasurable experiences to the user.

Matt McCormick (2001) has offered yet another argument according to which violent and degrading behavior in single-user VR can be construed as unethical. He argues that repeated engagement in such behavior erodes one's character and reinforces virtueless habits. He follows Aristotelian virtue ethics in arguing that this is bad because it makes it difficult for us to lead fulfilling lives, because as Aristotle has argued, a fulfilling life can only be lived by those who are of virtuous character. More generally, the argument can be made that the excessive use of single-user VR keeps one from leading a good life, even if one's actions in it are virtuous, because one invests into fictional worlds and fictional experiences that seem to fulfill one's desires but do not actually do so (Brey, forthcoming).

\section{Behavior in Multi-User VR}


Many unethical behaviors between persons in the real world can also occur in multi-user virtual environments. As discussed earlier in the section on reality and virtuality, there are two classes of real-world phenomena that can also exist in virtual form: institutional entities that derive their status from collective agreements, like money, marriage, and conversations, and certain physical and formal entities, like images and musical pieces, that computers are capable of physically realizing. Consequently, unethical behaviors involving such entities can also occur in VR, and it is possible for there to be real thefts, insults, deceptions, invasions of privacy, breaches of contract, or damage to property in virtual environments.

Immoral behaviors that cannot really happen in virtual environments are those that are necessarily defined over physically realized entities. For example, there can be real insult in virtual environments, but not real murders, because real murders are defined over persons in the physical world, and the medium of VR does not equip users with the power to kill persons in the physical world. It may, of course, be possible to kill avatars in VR, but these are of course not killings of real persons. It may also be possible to plan a real murder in VR, for example by using VR to meet up with a hitman, but this cannot then be followed up by the execution of a real murder in VR.

Even though virtual environments can be the site of real events with real consequences, they are often recognized as fictional worlds in which character merely play out roles. In such cases, even an insult may not be a real insult, in the sense of an insult made by a real person to another real person, because it may only have the status of an insult between two virtual characters. The insult is then only real in the context of the virtual world, but is not real in the real world. Ambiguities arise, however, because it will not always be clear when actions and events in virtual environments should be seen as fictional or real (Turkle, 1995). Users may assign different statuses to objects and events, and some users may identify closely with their avatar, so that anything that happens to their avatar also happens to them, whereas others may see their avatar as an object detached from themselves with which they do not identify closely. For this reason, some users may feel insulted when their avatar is insulted, whereas others will not feel insulted at all.

This ambiguity in the status of many actions and events in virtual worlds can lead to moral confusion as to when an act that takes place in VR is genuinely unethical and when it merely resembles a certain unethical act. The most famous case of this is the case of the "rape in cyberspace" reported by Julian Dibbell (1993). Dibbell reported an instance of a "cyberrape" in LambdaMOO, a text-only virtual environment in which users interact with user-programmable avatars. One user used a subprogram that took control of avatars and made them perform sex acts on each other. Users felt their characters were raped, and some felt that they themselves were indirectly raped or violated as well. But is it ever possible for someone to be raped through a rape of her avatar, or does rape require a direct violation of someone's body? Similar ambiguities exist for many other immoral practices in virtual environments, like adultery and theft. If it would constitute adultery when two persons were to have sex with each other, does it also constitute adultery when their avatars have sex? When a user steals virtual money or property from other users, should he be considered a thief in real life?

\section{Virtual Property and Virtual Economies}


For any object or structure found in a virtual world, one may ask the question: Who owns it? This question is already ambiguous, however, because there may both be virtual and real-life owners of virtual entities. For example, a user may be the owner of an island in a virtual world, but the whole world, including the island, may be owned by the company that has created it and permits users to act out roles in it. Users may also become creators of virtual objects, structures and scripted events, and some put in hundreds of hours of work into their creations. May they therefore also assert intellectual property rights to their creations? Or can the company that owns the world in which the objects are found and the software with which they were created assert ownership? What kind of framework of rights and duties should be applied to virtual property? (Burk, 2005).

The question of property rights in virtual worlds is further complicated by the emergence of so-called virtual economies. Virtual economies are economies that exist within the context of a persistent multi-user virtual world. Such economies have emerged in virtual worlds like Second Life and The Sims Online, and in massively multiplayer online role-playing games (MMORPGs) like Entropia Universe, World of Warcraft, Everquest and EVE Online. Many of these worlds have millions of users. Economies can emerge in virtual worlds if there are scarce goods and services in them for which users are willing to spend time, effort or money, if users can also develop specialized skills to produce such goods and services, if users are able to assert property rights on goods and resources, and if they can transfer goods and services between them.

Some economies in these worlds are primitive barter economies, whereas other make use of recognized currencies. Second Life, for example, makes use of the Linden Dollar (L\$) and Entropia Universe has the Project Entropia Dollar (PED), both of which have an exchange rate against real U.S. dollars. Users of these worlds can hence choose to acquire such virtual money by doing work in the virtual world (e.g., by selling services or opening a virtual shop) or by making money in the real world and exchanging it with virtual money. Virtual objects are now frequently traded for real money outside the virtual worlds that contain them, on online trading and auction sites like eBay. Some worlds also allow for the trade of land. In December 2006, the average price of a square meter of land in Second Life was L\$ 9.68 or U.S. \$ 0.014 (up from L\$ 6.67 in November), and over $36,000,000$ square meters were sold ${ }^{1}$ Users have been known to pay thousands of dollars for cherished virtual objects, and over $\$ 100,000$ for real estate.

The emergence of virtual economies in virtual environments raises the stakes for their users, and increases the likelihood that moral controversies ensue. People will naturally be more likely to act immorally if money is to be made or if valuable property is to be had. In one incident which took place in China, a man lent a precious sword to another man in the online game Legend of Mir 3, who then sold it to a third party. When the lender found out about this, he visited the borrower at his home and killed him. ${ }^{2}$ Cases have also been reported of Chinese sweatshop laborers who work day and night in conditions of practical slavery to collect resources in games like World of Warcraft and Lineage, which are then sold for real money.

\footnotetext{
${ }^{1}$ Source: https://secondlife.com/whatis/economy stats.php. Accessed 1/3/2007.

${ }^{2}$ Online gamer killed for selling cyber sword. ABC NewsOnline, March 30, 2005. http://www.abc.net.au/news/newsitems/200503/s1334618.htm.
} 
There have also been reported cases of virtual prostitution, for instance on Second Life, where users are paid to (use their avatar to) perform sex acts or to serve as escorts. There have also been controversies over property rights. On Second Life, for example, controversy ensued when someone introduced a program called CopyBot that could copy any item in the world. This program wreaked havoc on the economy, undermining the livelihood of thousands of business owners in Second Life, and was eventually banned after mass protests. ${ }^{3}$ Clearly, then, the emergence of virtual economies and serious investments in virtual property generates many new ethical issues in virtual worlds. The more time, money and social capital people invest in virtual worlds, the more such ethical issues will come to the front.

\section{The Ethics of Computer Games}

Contemporary computer and video games often play out in virtual environments or include computer simulations, as defined earlier. Computer games are nowadays mass media. A recent study shows that the average American 8- to 18-year old spends almost six hours per week playing computer games, and that $83 \%$ has access to a video game console at home (Rideout, Roberts and Foehr, 2005). Adults are also players, with four in ten playing computer games on a regular basis. ${ }^{4}$ In 2005, the revenue in the U.S. generated by the computer and game industry generated was over U.S. \$ 7 billion, far surpassing the film industry's annual box office results. ${ }^{5}$ Computer games have had a vast impact on youth culture, but also significantly influence the lives of adults. For these reasons alone, an evaluation of their social and ethical aspects is needed.

Some important issues bearing on the ethics of computer games have already been discussed in previous sections, and therefore will be covered less extensively here. These include, amongst others, ethical issues regarding biased and indecent representations; issues of responsibility and identity in the relation between avatars, users and bots; the ethics of behavior in virtual environments; and moral issues regarding virtual property and virtual economies. These issues, and the conclusions reached regarding them, all fully apply to computer games. The focus in this section will be on three important ethical questions that apply to computer games specifically: Do computer games contribute to individual well-being and the social good? What values should govern the design and use of computer games? And do computer games contribute to gender inequality?

\section{The Goods and Ills of Computer Games}

Are computer games a benefit to society? Many parents do not think so. They worry about the extraordinary amount of time their children spend playing computer games, and

\footnotetext{
${ }^{3}$ Linden bans CopyBot following resident protests. Reuters News, Wednesday November 15, 2006. http://secondlife.reuters.com/stories/2006/11/15/linden-bans-copybot-following-resident-protests/

${ }^{4}$ Poll: 4 in 10 adults play electronic games. MSNBC.com, May 8, 2006. http://www.msnbc.msn.com/id/12686020/

52006 Essential Facts about the Computer and Video Game Industry, Entertainment Software Association, 2006. http://www.theesa.com/archives/files/Essential\%20Facts\%202006.pdf
} 
about the excessive violence that takes place in many games. They worry about negative effects on family life, schoolwork and the social and moral development of their kids. In the media, there has been much negative reporting about computer games. There have been stories about computer game addiction and about players dying from exhaustion and starvation after playing video games for days on end. There have been stories about ultraviolent and otherwise controversial video games, and the ease by which children can gain access to them. The Columbine High School massacre, in 1999, in which two teenage students went out on a shooting rampage, was reported in the media to have been inspired by the video game Doom, and since then, other mass shootings have also been claimed to have been inspired by video games. Considerable doubt has been raised, therefore, as to whether computer games are indeed a benefit to society rather than a social ill.

The case against computer games tends to center on three perceived negative consequences: addiction, aggression and maladjustment. The perceived problem of addiction is that many gamers get so caught up in playing that their health, work or study, family life, and social relations suffer. How large this problem really is has not yet been adequately documented (though see Chiu, Lee and Huang, 2004). There clearly is a widespread problem, as there has been a worldwide emergence of clinics for video addicts in recent years. Not all hard-core gamers will be genuine addicts in the psychiatric sense, but many do engage in overconsumption, resulting in the neglect described above. The partners of adults who engage in such overconsumption sometimes called gamer widows, analogous to soccer widows, denoting that they have a relationship with a gamer who pays more attention to the game than to them.

Whereas there is no doubt that addiction to video games is a real social phenomenon, there is less certainty that playing video games can be correlated with increased aggression, as some have claimed. The preponderance of the evidence seems to indicate, however, that the playing of violent video games can be correlated with increases in aggression, including increases in aggressive thoughts, aggressive feeling, aggressive behaviors, and a desensitization to real-life violence, and a decrease in helpful behaviors (Carnagey, Anderson and Bushman, forthcoming; Bartholow, 2005).

However, some studies have found no such correlations, and present findings remain controversial. Whatever the precise relation between violent video games and aggression turns out to be, it is clear now that there is a huge difference between the way that children are taught to behave towards others by their parents and how they learn to behave in violent video games. This at least raises the question of how their understanding of and attitude towards violence and aggression is influenced by violent video games.

A third hypothesized ill of video games is that they cause individuals to be socially and cognitively slighted and maladjusted. This maladjustment is attributed in part to the neglect of studies and social relations due to an overindulgence into video games and to increased aggression levels from playing violent games. But it is also held to be due to the specific skills and understandings that users gain from video games. Children that play video games are exposed to conceptions of human relations and the workings of the world that have been designed into them by game developers. These conceptions have not been designed to be realistic or pedagogical, and often rely on stereotypes and simplistic modes of interaction and solutions to problems. It is therefore 
conceivable that children develop ideas and behavioral routines while playing computer games that leave much to be desired.

The case in favor of computer games begins with the observation that they are a new and powerful medium that brings users pleasure and excitement, and that allow for new forms of creative expression and new ways of acting out fantasies. Games moreover do not just cause social isolation, they can also stimulate social interaction. Playing multiplayer games is a social activity that involves interactions with other players, and that can even help solitary individuals find new friends. Computer games may moreover induce social learning and train social skills. This is especially true for role-playing games and games that involve verbal interactions with other characters. Such games let players experiment with social behavior in different social settings, and role-playing game can also make users intimately familiar with the point of view and experiences of persons other than themselves. Computer games have moreover been claimed to improve perceptual, cognitive and motor skills, for example by improving hand-eye coordination and improving visual recognition skills (Johnson, 2005; Green and Bavelier, 2003).

\section{Computer Games and Values}

It has long been argued in computer ethics that computer systems and software are not value-neutral but are instead value-laden (Nissenbaum, 1998; Brey, 2000). Computer games are no exception. Computer games may suggest, stimulate, promote or reward certain values while shunning or discouraging others. Computer games are value-laden, first of all, in the way they represent the world. As discussed, earlier, such representations may contain a variety of biases. They may, for example, promote racial and gender stereotypes (Chan, 2005; Ray, 2003), and they may contain implicit, biased assumptions about the abilities, interests or gender of the player. Simulation games like SimCity may suggest all kinds of unproven causal relations, for example correlations between poverty and crime, that may help shape attitudes and feed prejudices. Computer games may also be value-laden in the interactions that they make possible. They may, for example, be designed to make violent action the only solution to problems faced by a player. Computer games can also be value-laden in the storylines they suggest for players and in the feedback and rewards that are given. Some first-person shooters awards extra points, for example, for not killing innocent bystanders, whereas others instead award extra points for killing as many as possible.

A popular game like The Sims can serve to illustrate how values are embedded in games. The Sims is a game that simulates the everyday lives and social relationships of ordinary persons. The goal of characters in the game is happiness, which is attained through the satisfaction of needs like Hunger, Comfort, Hygiene and Fun. These needs can be satisfied through success in one's career, and through consumption and social interaction. As Miguel Sicart (2003) has argued, The Sims thus presents an idealized version of a progressive liberal consumer society in which the goal in life is happiness, gained by being a good worker and consumer. The team-based first-person shooter America's Army presents another example. This game is offered as a free download by the U.S. government, who uses it to stimulate U.S. army recruitment. The game is designed to give a positive impression of the U.S. army. Players play as servicemen who 
obey orders and work together to combat terrorists. The game claims to be highly realistic, yet it has been criticized for not showing certain realistic aspects of military life, such as collateral damage, harassment, and gore.

The question is how influential computer games actually are in influencing the values of players. The amount of psychological research done of this topic is still limited. However, psychological research on the effect of other media, such as television, has shown that it is very influential in affecting the value of media users, especially children. Since many children are avid consumers of computer games, there are reasons to be concerned about the values projected on them by such games. Children are still involved in a process of social, moral and cognitive development, and computer games seem to have an increasingly large role in this developmental process. Concern about the values embedded in video games therefore seems warranted. On the other hand, computer games are games, and therefore should allow for experimentation, fantasy, and going beyond socially accepted boundaries. The question is how games can support such social and moral freedom without also supporting the development of skewed values in younger players.

Players do not just develop values on the basis of the structure of the game itself, they also develop them by interacting with other players. Players communicate messages to each other about game rules and acceptable in-game behavior. They can respond positively or negatively to certain behaviors, and may praise or berate other players. In this way, social interactions in games may become part of the socialization of individuals and influence their values and social beliefs. Some of these values and norms may remain limited to the game itself, for example, norms governing the permissibility of cheating (Kimppa and Bissett, 2005). In some games, however, like massively multiplayer online role-playing games (MMORPG's), socialization processes are so complex as to resemble real life (Warner and Raiter, 2005), and values learned in such games may be applied to real life as well.

\section{Computer Games and Gender}

Games magazine and game advertisement foster the impression that computer games are a medium for boys and men. Most pictured gamers are male, and many recurring elements in images, such as scantily clad, big-breasted women, big guns and fast cars, seem to be geared toward men. The impression that computer games are mainly a medium for men is further supported by usage statistics. Research has consistently shown that fewer girls and women play computer games than boys and men, and those that do spend less time playing than men. According to research performed by Electronic Arts, a game developer, among teenagers only $40 \%$ of girls play computer games, compared to $90 \%$ of boys. Moreover, when they reach high school, most girls lose interest, whereas most boys keep playing. ${ }^{6}$ A study by the UK games trade body, the Entertainment and Leisure Publishers Association, found that in Europe, women gamers make up only a quarter of the gaming population. ${ }^{7}$

\footnotetext{
${ }^{6}$ Games industry is 'failing women'. BBC News, August 21, 2006. http://news.bbc.co.uk/2/hi/technology/5271852.stm

${ }^{7}$ Chicks and Joysticks. An Exploration of Women and Gaming. ELSPA White Paper, September 2004. www.elspa.com/assets/files/c/chicksandjoysticksanexplorationofwomenandgaming_176.pdf
} 
The question whether there is a gender bias in computer games is morally significant because it is a question about gender equality. If it is the case that computer games tend to be designed and marketed for men, then women are at an unfair disadvantage, as they consequently have less opportunity to enjoy computer games and their possible benefits. Among such benefits may be greater computer literacy, an important quality in today's marketplace. But is the gender gap between usage of computer games really the result of gender bias in the gaming industry, or could it be the case that women are simply less interested in computer games than men, regardless of how games are designed and marketed?

Most analysts hold that the gaming industry is largely to blame. They point to the fact that almost all game developers are male, and that there have been few efforts to develop games suitable for women. To appeal to women, it has been suggested, computer games should be less aggressive, because women have been socialized to be non-aggressive (Norris, 2004). It has also been suggested that women have a greater interest in multiplayer games, games with complex characters, games that contain puzzles, and games that are about human relationships. Games should also avoid assumptions that the player is male and avoid stereotypical representations of women. Few existing games contain good role models for women. Studies have found that most female characters in games have unrealistic body images and display stereotypical female behaviors, and that a disproportionate number of them are prostitutes and strippers. ${ }^{8}$

\section{Virtual Reality, Simulation and Professional Ethics}

In discussing issues of professional responsibility in relation to virtual reality systems and computer simulations, a distinction can be made between the responsibility of developers of such systems and that of professional users. Professional users can be claimed to have a responsibility to acquaint themselves with the technology and its potential consequences and to use it in a way that is consistent with the ethics of their profession. The responsibility of developers includes giving consideration to ethical aspects in the design process and engaging in adequate communication about the technology and its effects to potential users.

In the development of computer simulations, the accuracy of the simulation and its reliability as a foundation for decision-making in the real world are of paramount importance. The major responsibility of simulation professionals is therefore to avoid misrepresentations where they can and to adequately communicating the limitations of simulations to users (McLeod, 1983). These responsibilities are, indeed, a central ingredient in a recent code of ethics for simulationists adopted by a large number of professional organizations for simulationists (Ören et al., 2002). The responsibility for accuracy entails the responsibility to take proper precautions to ensure that modeling mistakes do not occur, especially when the stakes are high, and to inform users if inaccuracies do or may occur. It also entails the responsibility not to participate in intentional deception of users (e.g., embellishment, dramatization, or censorship).

\footnotetext{
${ }^{8}$ Fair Play: Violence, Gender and Race in Video Games. Children Now , December 2001. 36 pp. http://publications.childrennow.org/
} 
In Brey (1999), I have argued that designers of simulations and virtual environments also have a responsibility to incorporate proper values into their creations. It has been argued earlier that representations and interfaces are not value-free but may contain values and biases. Designers have a responsibility to reflect on the values and biases contained in their creations and to ensure that they do not violate important ethical principles. The responsibility to do this follows from the ethical codes that are in use in different branches of engineering and computer science, especially the principle that professional expertise should be used for the enhancement of human welfare. If technology is to promote human welfare, it should not contain biases and should regard the values and interests of stakeholders or society at large. Taking into account such values and avoiding biases in design cannot be done without a proper methodology. Fortunately, a detailed proposal for such a methodology has recently been made by Batya Friedman and her associates, and has been termed value-sensitive design (Friedman, Kahn and Borning, 2006).

Special responsibilities apply to different areas of applications for VR and computer simulations. The use of virtual reality in therapy and psychotherapy, for example, requires special consideration to principles of informed consent and the ethics of experimentation with human subjects (Wiederhold and Wiederhold, 2004). The computer and video game industry can be argued to have a special responsibility to consider the social and cultural impact of their products, given that they are used by a mass audience that includes children. Arguably, game developers should consider the messages that their products send to users, especially children, and should work to ensure that they develop and market content that is age-appropriate and that is more inclusive of all genders.

Virtual reality and computer simulation will continue to present new challenges for ethics, because new and more advanced applications are still being developed, and their use is more and more widespread. Moreover, as has been argued, virtual environments can mimic many of the properties of real life, and therefore contain many of the ethical dilemmas found in real life. It is for this reason that they will not just continue to present new ethical challenges for professional developers and users, but also for society at large.

\section{References}

Bartholow, B. (2005). Correlates and Consequences of Exposure to Video Game Violence: Hostile Personality, Empathy, and Aggressive Behavior. Personality and Social Psychology Bulletin, 31(11), 1573-1586.

Baudrillard, J. (1995). Simulacra and Simulation. Trans. S. Fraser. Ann Arbor, MI: University of Michigan Press.

Borgmann, A. (1999). Holding On to Reality: The Nature of Information at the Turn of the Millennium. University Of Chicago Press

Brey, P. (1998). New Media and the Quality of Life. Techné: Journal of the Society for Philosophy and Technology 3(1), 1-23.

Brey, P. (1999). The Ethics of Representation and Action in Virtual Reality. Ethics and Information Technology 1(1), 5-14.

Brey, P. (2000). Disclosive Computer Ethics. Computers and Society, 30(4), 10-16.

Brey, P. (2003). The Social Ontology of Virtual Environments. American Journal of Economics and Sociology 62(1), 269-282. 
Brey, P. (forthcoming). Theorizing the Cultural Quality of New Media, Techné. Research in Philosophy and Technology.

Burdea, G. and Coiffet, P. (2003). Virtual Reality Technology, $2^{\text {nd }}$ ed. John Wiley \& Sons.

Burk, D. (2005). Electronic Gaming and the Ethics of Information Ownership. International Review of Information Ethics 4, 39-45.

Carnagey, N., Anderson, C. and Bushman, B. (forthcoming). The effect of video game violence on physiological desensitization to real-life violence. Journal of Experimental Social Psychology.

Chan, D (2005). Playing with Race: The Ethics of Racialized Representations in E-Games. International Review of Information Ethics 4, 24-30.

Chiu, S., Lee, J. and Huang, D. (2004). Video Game Addiction in Children and Teenagers in Taiwan. Cyberpsychology \& Behavior 7(5), 571-581.

Dibbell, J. (1993). A Rape in Cyberspace. The Village Voice, December 21, 1993, 36-42. Reprinted in Reading Digital Culture, ed. D. Trend, Blackwell, 199-213.

Dreyfus, H. (2001). On the Internet. Routledge.

Ford, P. (2001). A further analysis of the ethics of representation in virtual reality: Multi-user environments. Ethics and Information Technology 3: 113-121.

Friedman, B., Kahn, P. H., Jr., \& Borning, A. 2006. Value Sensitive Design and Information Systems. In P. Zhang \& D. Galletta (eds.), Human-computer interaction in management information systems: Foundations (pp. 348-372). Armonk, New York; London, England: M.E. Sharpe, 348-72.

Green, C. S. and Bavelier, D. (2003). Action video game modifies visual selective attention. Nature 423, 29 May 2003, 534-537.

Heim, M. (1994). The Metaphysics of Virtual Reality. New York: Oxford University Press.

Johnson, S. (2005). Everything Bad Is Good for You: How Today's Popular Culture Is Actually Making Us Smarter. New York: Riverhead Books.

Kimppa, K. and Bissett, (2005). The Ethical Significance of Cheating in Online Computer Games. International Review of Information Ethics 4, 31-37.

Levy, N. (2002). Virtual child pornography: The Eroticization of Inequality. Ethics and Information Technology 4, 319-323.

McCormick, M. (2001). Is it wrong to play violent video games? Ethics and Information Technology 3(4), 277-287.

McLeod, J. (1983). Professional Ethics and Simulation. Proceedings of the 1983 Winter Simulation Conference. Ed. S. Roberts, J. Banks, B. Schmeiser, 371-3.

Nissenbaum, H. (1998). Values in the Design of Computer Systems. Computers and Society, March $1998,38-39$.

Norris. , K. (2004). Gender Stereotypes, Aggression, and Computer Games: An Online Survey of Women. Cyberpsychology \& Behavior 7(6), 714-27.

Ören, T.I., Elzas, M.S., Smit, I., and L.G. Birta (2002). A Code of Professional Ethics for Simulationists. Proceedings of the 2002 Summer Computer Simulation Conference, San Diego, CA, pp. 434-435.

Prosser, W. (1960). Privacy. California Law Review 48(4), 383-423.

Ray. S. (2003). Gender Inclusive Game Design: Expanding The Market. Charles River Media.

Rideout, V., Roberts, D. and Foehr, U. (2005). Generation M: Media in the Lives of 8-18 Year-olds. A Kaiser Family Foundation Study, March 2005. http://www.kff.org/entmedia/entmedia030905pkg.cfm

Sandin, P. 'Virtual Child Pornography and Utilitarianism.' Journal of Information, Communication \& Ethics in Society 2(4), 217-223, 2004.

Searle, J. (1995). The Construction of Social Reality. Cambridge, MA: MIT Press.

Sherman, W. and Craig, W. (2003). Understanding Virtual Reality. Interface, Application and Design. Morgan Kaufmann Publishers.

Sicart, M. (2003). Family Values: Ideology, Computer Games \& Sims. Level Up Conference Proceedings, Utrecht: University of Utrecht (CD-ROM).

Slater, M., Antley, M., Davison, A., Swapp, D., Guger, C., Barker, C., Pistrang, N. \& Sanchez-Vives, M. (2006). A Virtual Reprise of the Stanley Milgram Obedience Experiments. PLoS ONE, 1(1), e39 (open access)

Tabach-Bank, J. (2004). Missing the right of publicity boat: How Tyne v. Time Warner Entertainment Co. threatens to "Sink" the First Amendment. Loyola of Los Angeles Entertainment Law Review 24(2), 247-88.

Turkle, S. (1995). Life on the Screen. Identity in the Age of the Internet. Simon \& Schuster. 
Warner, D. and Raiter, M. (2005). Social Context in Massively-Multiplayer Online Games (MMOGs): Ethical Questions in Shared Space. International Review of Information Ethics 4, 47-52.

Wiederhold, B. and Wiederhold, M. (2004). Virtual Reality Therapy for Anxiety Disorders: Advances in Evaluation and Treatment. Washington, D.C.: American Psychological Association

Zhai, P. (1998). Get Real: A Philosophical Adventure in Virtual Reality. Rowman \& Littlefield Publishers. 